Research Article

\title{
Genetic Diversity, Antimicrobial Resistance Pattern, and Biofilm Formation in Klebsiella pneumoniae Isolated from Patients with Coronavirus Disease 2019 (COVID-19) and Ventilator- Associated Pneumonia
}

\author{
Asma Ghanizadeh, ${ }^{1}$ Maede Najafizade $\mathbb{D}^{1},{ }^{1}$ Somaye Rashki ${ }^{\mathbb{D}},{ }^{2}$ Zeynab Marzhoseyni, \\ and Mitra Motallebi $\mathbb{D i D}^{2,3}$ \\ ${ }^{1}$ Department of Infectious Disease, School of Medicine, Kashan University of Medical Sciences, Kashan, Iran \\ ${ }^{2}$ Department of Immunology and Microbiology, Faculty of Medicine, Kashan University of Medical Sciences, Kashan, Iran \\ ${ }^{3}$ Infectious Diseases Research Center, Kashan University of Medical Sciences, Kashan, Iran \\ Correspondence should be addressed to Mitra Motallebi; motallebi-m@kaums.ac.ir
}

Received 19 April 2021; Revised 2 July 2021; Accepted 6 December 2021; Published 24 December 2021

Academic Editor: Fu-Ming Tsai

Copyright ( $) 2021$ Asma Ghanizadeh et al. This is an open access article distributed under the Creative Commons Attribution License, which permits unrestricted use, distribution, and reproduction in any medium, provided the original work is properly cited.

\begin{abstract}
Introduction. Patients with acute respiratory distress syndrome caused by coronavirus disease 2019 (COVID-19) are at risk for superadded infections, especially infections caused by multidrug resistant (MDR) pathogens. Before the COVID-19 pandemic, the prevalence of MDR infections, including infections caused by MDR Klebsiella pneumoniae (K. pneumoniae), was very high in Iran. This study is aimed at assessing the genetic diversity, antimicrobial resistance pattern, and biofilm formation in $K$. pneumoniae isolates obtained from patients with COVID-19 and ventilator-associated pneumonia (VAP) hospitalized in an intensive care unit (ICU) in Iran. Methods. In this cross-sectional study, seventy K. pneumoniae isolates were obtained from seventy patients with COVID-19 hospitalized in the ICU of Shahid Beheshti hospital, Kashan, Iran, from May to September, 2020. K. pneumoniae was detected through the ureD gene. Antimicrobial susceptibility testing was done using the Kirby-Bauer disc diffusion method, and biofilm was detected using the microtiter plate assay method. Genetic diversity was also analyzed through polymerase chain reaction based on enterobacterial repetitive intergenic consensus (ERIC-PCR). The BioNumerics software (v. 8.0, Applied Maths, Belgium) was used for analyzing the data and drawing dendrogram and minimum spanning tree. Findings. K. pneumoniae isolates had varying levels of resistance to antibiotics meropenem (80.4\%), cefepime-aztreonampiperacillin/tazobactam (70\%), tobramycin (61.4\%), ciprofloxacin $(57.7 \%)$, gentamicin $(55.7 \%)$, and imipenem (50\%). Around $77.14 \%$ of isolates were MDR, and $42.8 \%$ of them formed biofilm. Genetic diversity analysis revealed 28 genotypes (E1-E28) and $74.28 \%$ of isolates were grouped into ten clusters (i.e., clusters A-J). Clusters were further categorized into three major clusters, i.e., clusters E, H, and J. Antimicrobial resistance to meropenem, tobramycin, gentamicin, and ciprofloxacin in cluster $\mathrm{J}$ was significantly higher than cluster $\mathrm{H}$, denoting significant relationship between ERIC clusters and antimicrobial resistance. However, there was no significant difference among major clusters $\mathrm{E}, \mathrm{H}$, and $\mathrm{J}$ respecting biofilm formation. Conclusion. K. pneumoniae isolates obtained from patients with COVID-19 have high antimicrobial resistance, and $44.2 \%$ of them have genetic similarity and can be clustered in three major clusters. There is a significant difference among clusters respecting antimicrobial resistance.
\end{abstract}

\section{Introduction}

Coronavirus disease 2019 (COVID-19) is a new emerging disease in human population. The World Health Organiza- tion introduced COVID-19 as a pandemic in March 11, 2020. The disease is caused by a virus called severe acute respiratory syndrome coronavirus 2 (SARS-CoV-2) $[1,2]$. The clinical manifestations of COVID-19 include fever, 
leukocytosis, severe hypoxemia, bilateral pulmonary infiltrates, multisystem inflammatory syndrome, and multiorgan failure. Five waves of COVID-19 outbreak have been reported in Iran so far [1].

The SARS-CoV-2 virus often affects ciliated cells in the alveolar epithelium and reduces their normal activities such as airway clearance. Subsequent gradual accumulation of fluid and residuals in the lung results in acute respiratory distress syndrome. Some afflicted patients need hospitalization in intensive care unit (ICU) to receive intensive care and mechanical ventilation [3].

Pulmonary infiltration and mechanical ventilation predispose patients with COVID-19 in ICU to secondary bacterial infections. Previous studies reported that $40 \%-86 \%$ of patients with COVID-19 receiving mechanical ventilation developed ventilator-associated pneumonia (VAP). The prevalence of VAP among patients in ICU varies according to their clinical conditions, ICU admission policies, and types of treatment $[1,4]$. The most common gram-negative microorganisms contributing to VAP are Escherichia coli, Klebsiella pneumoniae, Pseudomonas aeruginosa, and Acinetobacter baumannii [2]. However, there is limited information about microorganisms which cause VAP among patients with COVID-19 in ICU [5].

Klebsiella pneumoniae (K. pneumoniae) is one of the most common pathogens contributing to VAP in ICU in the United States and Middle East countries like Iran [6]. It is a highly prevalent gram-negative bacterium which causes lethal nosocomial infections throughout the world [7]. In recent years, nosocomial infections caused by multidrug resistant (MDR) strains of K. pneumoniae (MDRKp) have been a major public health concern. Several studies reported the high prevalence of nosocomial infections caused by $K$. pneumoniae strains which were resistant to third generation cephalosporins, aminoglycosides, and quinolones. Some MDRKp strains have changed to extensive drug resistance (XDR) strains. These strains are usually a major threat to patients with serious health conditions such as COVID-19 due to the ineffectiveness of treatments against them and their high mortality rate [8-10].

$K$. pneumoniae can form biofilm which is an extracellular matrix consisted of proteins, exopolysaccharides, deoxyribonucleic acid (DNA), and lipopeptides and protects bacteria against antibiotics $[11,12]$. Biofilm facilitates bacterial attachment to living and nonliving surfaces, prevents the penetration of antibiotics, and reduces the effects of antibiotics [13]. Thick biofilm formation is also observed in VAP and among colonized bacteria in endotracheal tube and ventilator circuit. These bacteria are mostly resistant to antibiotics due to their physical isolation from blood circulation, their ability to easily adapt to oxygen deprivation and $\mathrm{pH}$ changes, and inability of antibiotics to penetrate the full depth of the biofilm [14].

Moreover, K. pneumoniae has some virulence factors, such as capsular polysaccharides, lipopolysaccharides, types 1 and 3 fimbriae, outer membrane proteins, and factors determining iron acquisition and nitrogen source, and uses them for survival and evasion from the immune system of the body $[15,16]$. Adequate knowledge about biofilm and virulence factors is critical for the effective management of nosocomial infections [17].

Bacteria have different genetic characteristics which determine their virulence and behaviors. Determining the genetic diversity of bacteria helps determine the most prevalent bacterial strains in a given setting, determine the sources of infections, and determine the best preventive measures and infection control policies for that setting [18, 19]. Typing methods are usually used for determining genetic diversity, assessing epidemiologic concordance, and determining the sources of infection. However, the reproducibility, stability, discriminatory power, and epidemiologic concordance of these methods should be assessed before their use [20].

Enterobacterial repetitive intergenic consensus (ERIC) is one of the repetitive elements which vary in different bacterial genomes in terms of pattern and number. Polymerase chain reaction (PCR) based on ERIC (ERIC-PCR) has successfully been used for the genotyping, strain diversity assessment, population analysis, and epidemiological assessments of different microbial pathogens and determining their taxonomy and phylogenetic relatedness [21]. Compared with other typing methods such as ribotyping, Pulse Field Gel Electrophoresis, and Multilocus Sequence Typing, ERIC-PCR is considered as a faster, more reliable, and more cost-effective technique for the molecular typing and the genetic diversity assessment of the Enterobacteriaceae family [22].

Kashan is a city in the center of Iran. Before the onset of the COVID-19 pandemic, high prevalence of MDRKp infections had been reported in the leading hospital of the city [23]. However, there were limited data about the microbiological characteristics of $K$. pneumoniae among patients with COVID-19 in the ICU of this hospital. The present study was conducted to narrow this gap. The study is aimed at assessing the genetic diversity, antimicrobial resistance pattern, and biofilm formation in $K$. pneumoniae isolates obtained from patients with COVID-19 and VAP in ICU.

\section{Materials and Methods}

2.1. Design. This descriptive study was conducted during the second wave of COVID-19 in Iran, i.e., from May to September, 2020.

2.2. Sample Collection. Seventy K. pneumoniae isolates were obtained from seventy patients with COVID-19 hospitalized in the ICU of Shahid Beheshti hospital, Kashan, Iran. This hospital is the main COVID-19 care center in Kashan city in the center of Iran. All K. pneumoniae isolates were obtained through sampling from patients' tracheal secretions and then were immediately cultured in an appropriate culture medium in the laboratory of the Microbiology Department of Kashan University of Medical Sciences, Kashan, Iran. Data on participants' demographic characteristics were collected from their medical records.

2.3. Ethical Considerations. The Ethics Committee of Kashan University of Medical Sciences, Kashan, Iran, approved this study (code: IR.KAUMS.MEDNT.REC.1399.034). Sampling 
and data collection were performed under the supervision of this committee. All participants provided written informed consent.

2.4. K. pneumoniae Detection. Phenotypic detection of $K$. pneumoniae to the species level was performed based on biochemical reactions, including reaction on $\mathrm{SH} 2 /$ indole/motility (SIM) medium, triple sugar iron (TSI) agar, urease production on urea agar, growth on Simmons' citrate agar medium, methyl red/Vogues-Proskauer (MR/VP), and ornithine decarboxylase (OD) test [23]. Before analysis, all isolates were cultured on brain-heart infusion agar (CONDA, Spain) and stored in tryptic soy broth (TSB) (CONDA, Spain) with $15 \%$ glycerol at a temperature of $-70^{\circ} \mathrm{C}$. Then, the PCR method was used to confirm K. pneumoniae isolates through detecting the ure $\mathrm{D}$ gene. This gene is responsible for urea hydrolysis. The forward primer $5^{\prime}$-CCCGTTTTACC CGGAAGAAG- $3^{\prime}$ and the reverse primer $5^{\prime}$-GGAAAG AAGATGGCATCCTGC- $3^{\prime}$ were employed for the amplification of 243 base pairs (bp) of the ureD gene [24]. PCR was performed in a final reaction volume of $25 \mu \mathrm{L}$ as follows: initial denaturation at $95^{\circ} \mathrm{C}$ for three minutes, denaturation at $95^{\circ} \mathrm{C}$ for thirty thirty-second cycles, annealing at $95^{\circ} \mathrm{C}$ for 45 seconds, elongation at $72^{\circ} \mathrm{C}$ for sixty seconds, and final extension at $72^{\circ} \mathrm{C}$ for sixty seconds. The final products of PCR were electrophoresed on agarose gel.

2.5. Antimicrobial Susceptibility Testing. Antimicrobial susceptibility testing was performed for all detected K. pneumoniae isolates using the Kirby-Bauer disc diffusion method based on the guidelines of the Clinical and Laboratory Standards Institute (CLSI) [25]. Accordingly, the antimicrobial resistance pattern of $K$. pneumoniae isolates was identified with respect to the following ten antibiotics: piperacillin/tazobactam (PTZ, 100/10 $\mu \mathrm{g})$, cefepime (CPM, $30 \mu \mathrm{g})$, imipenem (IMP, $10 \mu \mathrm{g}$ ), meropenem (Mer, $10 \mu \mathrm{g})$, gentamicin $(\mathrm{GM}, 10 \mu \mathrm{g})$, tobramycin (TOB, $10 \mu \mathrm{g}$ ), aztreonam (AZT, $30 \mu \mathrm{g})$, ciprofloxacin (CIP, $5 \mu \mathrm{g})$, polymyxin B $(300 \mu \mathrm{g})$, and colistin (CST, $10 \mu \mathrm{g})$. K. pneumoniae isolates which were resistant to three or more antimicrobial categories were considered as MDR [26]. Escherichia coli ATCC 25922 was used as control.

2.6. Biofilm Detection Using the Microtiter Plate Assay (MTP) Method. Biofilm was detected through the MTP method. MTP is a quantitative method to detect biofilm using a microplate reader. An overnight culture in the TSB culture medium was performed for each isolate at a temperature of $37^{\circ} \mathrm{C}$. Then, a bacterial suspension in TSB was prepared and adjusted to $0.5 \mathrm{McF}$ arland $\left(1.5 \times 10^{8} \mathrm{cfu} / \mathrm{ml}\right)$. This suspension was tenfold diluted to the concentration of $5 \times$ $10^{5}(\mathrm{cfu} / \mathrm{ml})$, and then, $200 \mu \mathrm{L}$ bacterial suspension was inoculated into 96-well flat-bottomed sterile microplate. Negative control wells containing $200 \mu \mathrm{L}$ TSB were also included in each test. Incubation at a temperature of $37^{\circ} \mathrm{C}$ was performed for 24 hours. After that, wells were gently washed three times using $200 \mu \mathrm{L}$ of distilled water, dried, and fixed using 99\% methanol during twenty minutes. Thereafter, biofilm mass was stained for fifteen minutes using $200 \mu \mathrm{L}$ of $0.1 \%$ crystal violet dye. After air drying the wells, the dye of the biofilm which had lined the walls of the microplate was resolubilized using 5\% isopropanol acid. Finally, the microplate was spectrophotometrically measured using a microplate reader at a wave length of $570 \mathrm{~nm}$ [27]. The optical density cutoff (ODc) was assigned as "an average OD of negative controls $+(3 \times$ standard deviation of negative controls)." Isolates were categorized respecting biofilm formation as follows: isolates with an OD equal to or less than ODc were considered as having no biofilm formation; isolates with an OD between ODc and 2ODc were categorized as weak biofilm producer; isolates with an $\mathrm{OD}$ between $2 \mathrm{ODc}$ and $4 \mathrm{ODc}$ were categorized as moderate biofilm producer; isolates with an OD more than $4 \mathrm{OD}$ were categorized as strong biofilm producer [27]. Each test was performed three times. In biofilm detection through the MTP method, PAO1 was used as positive control and TSB with $1 \%$ glucose was used as negative control.

2.7. ERIC-PCR. For epidemiologic typing, genomic DNA was extracted from bacterial cells through the method proposed by Purighalla et al. [28], and then, ERIC-PCR was performed for assessing genetic similarity among bacterial isolates. ERIC-PCR reactions were performed as previously described by Veresalovi et al. [29] using the primer ERIC1R $\left(5^{\prime}\right.$-ATG TAA GCT CCT GGG GAT TCAC- $\left.3^{\prime}\right)$ and the primer ERIC2 ( $5^{\prime}$-AAG TAA GTG ACT GGG GTG AGC G-3') (Metabion, Germany). PCR amplification was done using a mixture of $18 \mu \mathrm{l}$ of sterile distilled water, $2.5 \mu \mathrm{l}$ of $10 \times$ PCR buffer, $1 \mu \mathrm{l}$ of 10 molar dNTP, $1 \mu \mathrm{l}$ of each primer, $0.5 \mu \mathrm{l}$ of Taq polymerase, and $1 \mu \mathrm{l}$ of template DNA, i.e., a total volume of $25 \mu \mathrm{l}$ per reaction. PCR reaction consisted of an initial denaturation at $95^{\circ} \mathrm{C}$ for three minutes and then 35 thermal cycles consisting of denaturation at $94^{\circ} \mathrm{C}$ for one minute, annealing at $48^{\circ} \mathrm{C}$ for one minute, and final extension at $72^{\circ} \mathrm{C}$ for two minutes and at $72^{\circ} \mathrm{C}$ for five minutes.

2.8. ERIC Analysis. The BioNumerics software (v. 8.0, Applied Maths, Belgium) was used for band profile analysis. This version of the software has the options for analyzing and interpreting phenotypic and genotypic data such as the data obtained in gel- and sequence-based typing methods. For dendrogram construction, genetic similarity analysis was performed using Unweighted Pair Group Mean Method with Arithmetic mean (UPGMA), Dice similarity coefficient, and $1 \%$ band position tolerance. Only bands which sized $100-3000 \mathrm{bp}$ according to the ladder were considered for analysis. The BioNumerics software creates groups with specific similarity range. The difference among band patterns was depicted using numbers over lines in dendrogram (Figure 1(a)) or numbers between isolates in minimum spanning tree (MST) (Figure 1(b)). Based on band analysis, isolates with a genetic similarity of $80 \%$ or greater were grouped into an ERIC type, while isolates without such similarity were considered as separate one-isolate ERIC type. ERIC types were named E1-En. The criteria for ERIC clustering in the BioNumerics software were a difference of $20 \%$ or less and the presence of more than one isolate in 


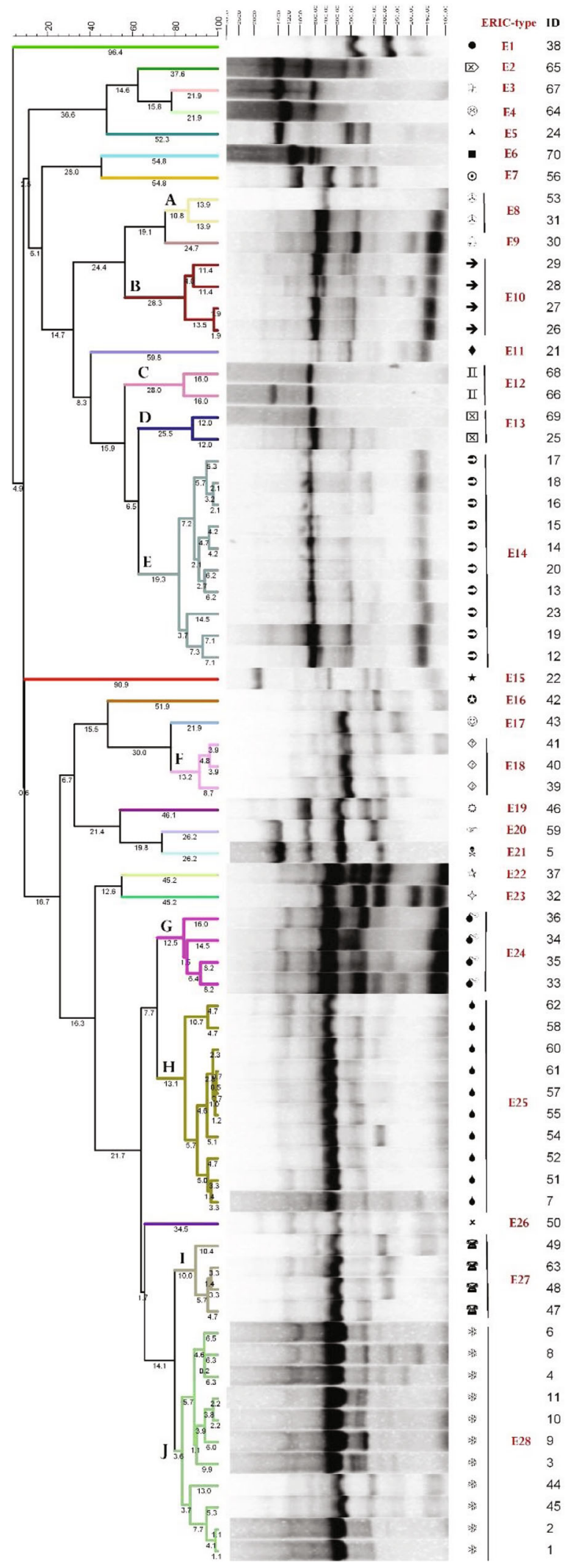

(a)

Figure 1: Continued. 


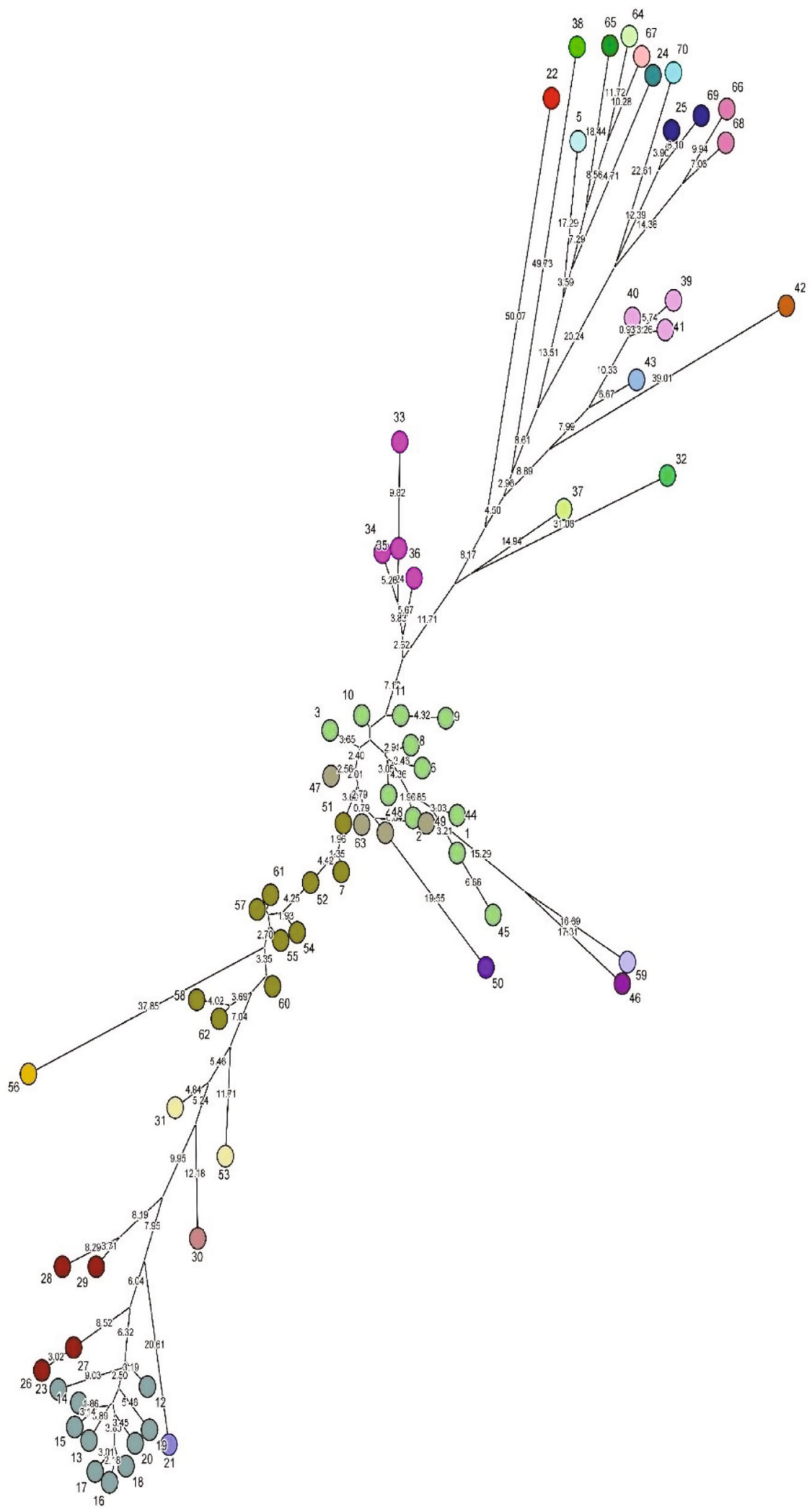

(b)

FIgURE 1: (a) Dendrogram. K. pneumoniae isolates with a genetic similarity of at least $80 \%$ were grouped into ten ERIC types, namely, A-J. (b) MST. Clusters were determined using the UPGMA method and Dice similarity coefficient. Numbers at the tip of each branch represent isolate numbers, and numbers over the lines show genetic difference between the isolates. 
each cluster (A-J). Finally, MST was generated in the software (Figure 1(b)).

\section{Statistical Analysis}

Data were analyzed using the Fisher's exact and the Chisquare tests. $P$ values less than 0.05 were considered statistically significant. Data analysis was performed using the SPSS software (v. 16.0).

\section{Findings}

In total, seventy tracheal $K$. pneumoniae isolates were obtained from patients with COVID-19 and VAP in ICU. Thirty-six isolates were from male patients, and 34 were from female patients. The mean of patients' age was $65.7 \pm$ 10.5 years (Table 1 ).

Antimicrobial susceptibility testing showed that K. pneumoniae isolates had varying levels of resistance to antibiotics Mer (80.4\%), CPM-AZT-PTZ (70\%), TOB (61.4\%), CIP (57.7\%), GM (55.7\%), and IMI (50\%). Table 1 shows antimicrobial resistance pattern of $K$. pneumoniae for ten tested antibiotics. All $K$. pneumoniae isolates were sensitive to polymyxin B and colistin, while $77.14 \%$ of isolates were MDRKp.

Findings showed that $42.8 \%$ of $K$. pneumoniae isolates formed biofilm. Biofilm analysis showed that $2.8 \%$ of isolates were strong biofilm producer $(n=2), 31.4 \%$ of them were moderate biofilm producer $(n=22), 8.5 \%$ of them were weak biofilm producer $(n=6)$, and the remaining $57.1 \%$ of them did not form biofilm $(n=40)$ (Table 1$)$.

Genetic similarity analysis through ERIC-PCR revealed 28 genotypes among the seventy $K$. pneumoniae isolates (E1-E28). These ERIC types consisted of ten genotypes with a genetic similarity of more than $80 \%$ and eighteen isolates with a genetic similarity of less than $80 \%$. Each of these eighteen isolates was placed in a separate ERIC type (Figure 1(a)). During MST and dendrogram analysis, most isolates $(n=52)$ were placed in ten clusters (i.e., clusters $\mathrm{A}-\mathrm{J})$. The clusters were further categorized into three major clusters, i.e., clusters $\mathrm{E}, \mathrm{H}$, and J, with ten, ten, and eleven isolates, respectively. Moreover, 21 isolates were placed in seven minor clusters, namely, clusters A, B, C, D, F, G, and I, each with 2-4 isolates (Figure 1).

The analysis of antimicrobial resistance pattern showed that the most common patterns among $K$. pneumoniae isolates were, respectively, $\mathrm{Mer}+\mathrm{CPM}+\mathrm{TOB}+\mathrm{AZT}+\mathrm{IMI}+\mathrm{GM}$ $+\mathrm{CIP}+\mathrm{PTZ}$ and Mer+CPM+AZT+IMI+GM+CIP+PTZ, while the most common patterns in the three major clusters of $\mathrm{E}, \mathrm{H}$, and $\mathrm{J}$ were, respectively, Mer+CPM+TOB+AZT $+\mathrm{IMI}+\mathrm{GM}+\mathrm{CIP}+\mathrm{PTZ}, \mathrm{Mer}+\mathrm{TOB}+\mathrm{CIP}$, and Mer+CPM $+\mathrm{AZT}+\mathrm{IMI}+\mathrm{GM}+\mathrm{CIP}+\mathrm{PTZ}$. Comparing the results of antimicrobial susceptibility tests in the major clusters $\mathrm{E}$ and $\mathrm{J}$ showed that antimicrobial resistance to Mer, TOB, CIP, and GM in cluster $\mathrm{J}$ was significantly greater than cluster $\mathrm{E}$ $(P<0.05)$. Moreover, antimicrobial resistance to TOB in cluster $\mathrm{H}$ was significantly greater than cluster E. However, there was no significant difference between clusters $J$ and $\mathrm{H}$ respecting antimicrobial resistance (Table 2 ). In molecular genotyping, there was no correlation between ERIC types and biofilm formation ability.

\section{Discussion}

This study assessed the genetic diversity, antimicrobial resistance pattern, and biofilm formation in K. pneumoniae isolates obtained from patients with COVID-19 and VAP in ICU. Most K. pneumoniae isolates in this study showed MDR patterns (77.14\%) and were resistant to routinely used antibiotics for treating $K$. pneumoniae infections such as beta-lactams, aminoglycosides, and quinolones. Study results also revealed the high prevalence of antimicrobial resistance among $K$. pneumoniae isolates. The high prevalence of MDRKp infection in the present study was in line with the findings of previous studies $[30,31]$. For example, two studies in Iran before the COVID-19 pandemic reported the high prevalence of $K$. pneumoniae among patients with VAP in ICU $[23,32]$. MDR strains cause treatment failure and hence are associated with higher mortality rate than non-MDR strains among patients with COVID-19 in ICU. VAP caused by MDR pathogens can increase mortality rate among patients in ICU by $60 \%$ [9]. Many different factors can affect the emergence and spread of antimicrobial resistance, acquisition of antimicrobial resistance genes, and transmission of these genes. These factors include healthcare exposure (such as hospitalization), use of medical devices, shortage of diagnostic equipment, lack of efficient surveillance systems, immunosuppression, travel to areas with high endemicity of MDR bacteria, no use of new antimicrobial treatments, use of antibiotics in agriculture and animal food products, and extensive and inappropriate use of antibiotics in hospital wards [33]. These factors might also have contributed to the high prevalence of antimicrobial resistance in the present study. Inappropriate use of antibiotics is a leading cause of antimicrobial resistance. International reports show that despite the low risk of bacterial infection and no necessity of antibiotic therapy for mild to moderate cases of viral infections [34], around $70 \%$ of hospitalized patients with COVID-19 receive broad-spectrum antibiotics as prophylaxis [35].

The high prevalence of antimicrobial resistance to antibiotics Mer and CIP is an important finding because these were the most commonly used antibiotics in the study setting for the management of K. pneumoniae infections. As $K$. pneumoniae is a major cause of VAP in ICU, its resistance to these antibiotics makes treatment difficult and causes challenges for the management of VAP among patients with COVID-19 in ICU. The mechanisms of resistance to carbapenems such as Mer among K. pneumoniae include changes in membrane permeability, production of wide-spectrum beta-lactamases, changes in porins, and expression of the efflux system genes [36]. Moreover, studies showed that the most important mechanism of K. pneumoniae resistance to fluoroquinolones such as CIP is plasmid-mediated horizontal gene transfer $[37,38]$. Our findings also revealed the high prevalence of resistance to GM among K. pneumoniae isolates. Previous studies also reported acquired resistance to aminoglycosides such as GM among both gram-negative 
TABle 1: Characteristics of patients and K. pneumoniae isolates.

\begin{tabular}{|c|c|c|c|c|c|c|c|}
\hline \multirow{2}{*}{ No. } & \multicolumn{2}{|c|}{ Patients } & \multicolumn{5}{|c|}{ K. pneumoniae isolates } \\
\hline & Gender & Age (year) & Antimicrobial resistance pattern & MDR & Biofilm formation & ERIC type & Cluster \\
\hline 1 & Female & 71 & Mer, CPM, TOB, AZT, GM, CIP, PTZ & + & No & E28 & $\mathrm{J}$ \\
\hline 2 & Female & 50 & Mer, CPM, TOB, AZT, IMI, GM, CIP, PTZ & + & No & E28 & $\mathrm{J}$ \\
\hline 3 & Male & 56 & Mer, CPM, TOB, AZT, IMI, GM, CIP, PTZ & + & No & E28 & $\mathrm{J}$ \\
\hline 4 & Male & 78 & Mer, CPM, TOB, AZT, IMI, GM, CIP, PTZ & + & No & E28 & $\mathrm{J}$ \\
\hline 5 & Male & 59 & - & - & No & $\mathrm{E} 21$ & - \\
\hline 6 & Female & 69 & Mer, CPM, TOB, AZT, GM, CIP, PTZ & + & No & E28 & $\mathrm{J}$ \\
\hline 7 & Female & 72 & Mer & - & No & E25 & $\mathrm{H}$ \\
\hline 8 & Male & 66 & Mer, CPM, TOB, AZT, IMI, CIP, PTZ & + & No & E28 & $\mathrm{J}$ \\
\hline 9 & Female & 49 & Mer, CPM, AZT, CIP, PTZ & + & Moderate & E28 & $\mathrm{J}$ \\
\hline 10 & Female & 81 & Mer, CPM, TOB, AZT, IMI, GEM, CIP, PTZ & + & No & E28 & $\mathrm{J}$ \\
\hline 11 & Female & 75 & Mer, CPM, AZT, GM, CIP, PTZ & + & No & E28 & $\mathrm{J}$ \\
\hline 12 & Male & 53 & Mer, CPM, AZT, IMI, CIP, PTZ & + & Moderate & E14 & $\mathrm{E}$ \\
\hline 13 & Male & 52 & Mer, CPM, AZT, IMI, GM, CIP, PTZ & + & Weak & E14 & $\mathrm{E}$ \\
\hline 14 & Male & 65 & Mer, CPM, TOB, AZT, IMI, GM, CIP, PTZ & + & No & E14 & $\mathrm{E}$ \\
\hline 15 & Male & 78 & Mer, CPM, AZT, IMI, GM, CIP, PTZ & + & Weak & E14 & $\mathrm{E}$ \\
\hline 16 & Male & 61 & - & - & No & E14 & $\mathrm{E}$ \\
\hline 17 & Male & 64 & - & - & Moderate & E14 & $\mathrm{E}$ \\
\hline 18 & Female & 80 & Mer, CPM, AZT, IMI, GM, CIP, PTZ & + & No & E14 & $\mathrm{E}$ \\
\hline 19 & Female & 79 & Mer, CPM, TOB, AZT, IMI, GM, CIP, PTZ & + & No & E14 & $\mathrm{E}$ \\
\hline 20 & Male & 73 & - & - & Moderate & E14 & $\mathrm{E}$ \\
\hline 21 & Female & 63 & Mer, CPM & - & Moderate & E11 & - \\
\hline 22 & Female & 46 & Mer, CPM, AZT, IMI, CIP, PTZ & + & No & E15 & - \\
\hline 23 & Female & 55 & - & - & Moderate & E14 & $\mathrm{E}$ \\
\hline 24 & Male & 58 & - & _ & Moderate & E5 & - \\
\hline 25 & Female & 61 & - & _ & Moderate & E13 & $\mathrm{D}$ \\
\hline 26 & Male & 51 & Mer, CPM, TOB, AZT, IMI, GM, CIP, PTZ & + & No & E10 & $\mathrm{B}$ \\
\hline 27 & Male & 76 & Mer, CPM, TOB, AZT, IMI, GM, CIP, PTZ & + & No & E10 & B \\
\hline 28 & Male & 79 & Mer & _ & Moderate & E10 & $\mathrm{B}$ \\
\hline 29 & Female & 67 & Mer, CPM, TOB, AZT, GM, PTZ & + & No & E10 & $\mathrm{B}$ \\
\hline 30 & Female & 58 & CPM, TOB, AZT, GM, CIP & + & No & E9 & - \\
\hline 31 & Female & 59 & TOB, CIP, PTZ & + & No & E8 & A \\
\hline 32 & Male & 80 & Mer, CPM, TOB, AZT, IMI, GM, CIP, PTZ & + & No & E23 & - \\
\hline 33 & Male & 77 & Mer, CPM, TOB, AZT, GM, CIP & + & No & E24 & G \\
\hline 34 & Male & 49 & Mer, CPM, TOB, AZT, IMI, GM, CIP, PTZ & + & Moderate & E24 & G \\
\hline 35 & Male & 52 & Mer, CPM, TOB, AZT, IMI, GM, CIP, PTZ & + & Strong & E24 & G \\
\hline 36 & Male & 54 & Mer, CPM, TOB, AZT, IMI, GM, CIP, PTZ & + & Moderate & E24 & G \\
\hline 37 & Male & 73 & Mer, CPM, TOB, AZT, IMI, GM, CIP, PTZ & + & No & E22 & - \\
\hline 38 & Male & 67 & Mer, CPM, TOB, AZT, GM, CIP, PTZ & + & Moderate & E1 & - \\
\hline 39 & Male & 61 & Mer, CPM, AZT, IMI, CIP, PTZ & + & Moderate & E18 & $\mathrm{F}$ \\
\hline 40 & Female & 73 & Mer & - & Weak & E18 & $\mathrm{F}$ \\
\hline 41 & Female & 70 & Mer & - & Moderate & E18 & $\mathrm{F}$ \\
\hline 42 & Female & 84 & PTZ & - & Weak & E16 & - \\
\hline 43 & Male & 50 & Mer, CPM, TOB, AZT, IMI, GM, CIP, PTZ & + & No & E17 & - \\
\hline 44 & Male & 72 & Mer, CPM, TOB, AZT, IMI, GM, CIP, PTZ & + & Moderate & E28 & $\mathrm{J}$ \\
\hline 45 & Female & 74 & Mer, TOB, GM, CIP & + & No & E28 & $\mathrm{J}$ \\
\hline 46 & Male & 60 & Mer, CPM, TOB, AZT, IMI, GM, CIP, PTZ & + & Moderate & E19 & - \\
\hline 47 & Female & 64 & Mer, CPM, TOB, AZT, IMI, GM, CIP, PTZ & + & Moderate & E27 & I \\
\hline 48 & Male & 56 & Mer, CPM, TOB, AZT, IMI, GM, CIP, PTZ & + & Moderate & E27 & I \\
\hline
\end{tabular}


TABLE 1: Continued.

\begin{tabular}{|c|c|c|c|c|c|c|c|}
\hline \multirow{2}{*}{ No. } & \multicolumn{2}{|c|}{ Patients } & \multicolumn{5}{|c|}{ K. pneumoniae isolates } \\
\hline & Gender & Age (year) & Antimicrobial resistance pattern & MDR & Biofilm formation & ERIC type & Cluster \\
\hline 49 & Male & 55 & Mer, CPM, AZT, IMI, GM, CIP, PTZ & + & No & E27 & I \\
\hline 50 & Male & 71 & Mer, TOB & - & Moderate & E26 & - \\
\hline 51 & Female & 77 & Mer, CPM, TOB, AZT, IMI, GM, CIP, PTZ & + & No & E25 & $\mathrm{H}$ \\
\hline 52 & Female & 83 & Mer, CPM, AZT, IMI, GM, CIP, PTZ & + & No & E25 & $\mathrm{H}$ \\
\hline 53 & Female & 48 & CPM, AZT, CIP, PTZ & + & No & E8 & A \\
\hline 54 & Female & 74 & Mer, CPM, TOB, IMI, CIP, PTZ & + & No & E25 & $\mathrm{H}$ \\
\hline 55 & Male & 66 & Mer, TOB, CIP & + & Moderate & E25 & $\mathrm{H}$ \\
\hline 56 & Male & 58 & Mer, CPM, TOB, AZT, CIP, PTZ & + & Weak & E7 & - \\
\hline 57 & Female & 68 & Mer, TOB, CIP & + & No & E25 & $\mathrm{H}$ \\
\hline 58 & Female & 69 & Mer, CPM, TOB, AZT, IMI, GM, CIP, PTZ & + & No & E25 & $\mathrm{H}$ \\
\hline 59 & Male & 84 & Mer, CPM, TOB, AZT, GM, CIP & + & Moderate & E20 & - \\
\hline 60 & Female & 79 & CPM, AZT & - & No & E25 & $\mathrm{H}$ \\
\hline 61 & Male & 70 & Mer, CPM, AZT, CIP, PTZ & + & No & E25 & $\mathrm{H}$ \\
\hline 62 & Male & 63 & Mer, TOB, AZT, IMI, GM, CIP, PTZ & + & No & E25 & $\mathrm{H}$ \\
\hline 63 & Female & 67 & Mer, TOB, CIP, PTZ & + & No & $\mathrm{E} 27$ & I \\
\hline 64 & Female & 81 & Mer, CPM, TOB, AZT, PTZ & + & Strong & $\mathrm{E} 4$ & - \\
\hline 65 & Male & 48 & Mer, TOB, AZT, IMI, CIP, PTZ & + & Weak & E2 & - \\
\hline 66 & Male & 62 & Mer, CPM, TOB, AZT, IMI, GM, CIP, PTZ & + & No & E12 & $\mathrm{C}$ \\
\hline 67 & Male & 79 & Mer, CPM, TOB, AZT, GM, CIP, PTZ & + & No & E3 & - \\
\hline 68 & Female & 73 & Mer, CPM, TOB, AZT, IMI, GM, CIP, PTZ & + & No & E12 & $\mathrm{C}$ \\
\hline 69 & Female & 71 & Mer, CPM, AZT, IMI, GM, CIP, PTZ & + & No & E13 & $\mathrm{D}$ \\
\hline 70 & Female & 65 & TOB, CIP & - & Moderate & E6 & - \\
\hline
\end{tabular}

TABLE 2: Comparing the three major clusters of $K$. pneumoniae isolates obtained from patients with COVID-19.

\begin{tabular}{|c|c|c|c|c|c|c|c|c|}
\hline \multirow{2}{*}{ Cluster } & \multicolumn{8}{|c|}{ Antibiotic } \\
\hline & Mer & $\mathrm{CPM}$ & TOB & AZT & IMI & GEM & CIP & PTZ \\
\hline $\mathrm{J}(n=11)(\%)$ & $11(100)$ & $10(90.9)$ & $9(81.8)$ & $10(90.9)$ & $6(54)$ & $9(81.8)$ & $11(100)$ & $10(90.9)$ \\
\hline $\mathrm{H}(n=10)(\%)$ & $9(90)$ & $5(50)$ & $6(60)$ & $6(60)$ & $5(50)$ & $4(40)$ & $8(80)$ & $6(60)$ \\
\hline $\mathrm{E}(n=10)(\%)$ & $6(60)$ & $6(60)$ & $0(0)$ & $6(60)$ & $6(60)$ & $0(0)$ & $6(60)$ & $6(60)$ \\
\hline \multirow{3}{*}{$P$ value } & $0.4762^{\#}$ & $0.635^{\#}$ & $0.3615^{\#}$ & $0.0635^{\#}$ & $1^{\#}$ & $0.0805^{\#}$ & $0.2143^{\#}$ & $0.1486^{\#}$ \\
\hline & $0.0351^{\wedge}$ & $0.1486^{\wedge}$ & $\leq 0.001^{\wedge}$ & $0.0635^{\wedge}$ & $1^{\wedge}$ & $\leq 0.001^{\wedge}$ & $0.0351^{\wedge}$ & $0.1486^{\wedge}$ \\
\hline & $0.3034^{*}$ & $1^{*}$ & $0.0108^{*}$ & $1^{*}$ & $1^{*}$ & $0.0902^{*}$ & $0.6285^{*}$ & $1^{*}$ \\
\hline
\end{tabular}

${ }^{\#}$ Comparison between clusters J and H. ${ }^{\wedge}$ Comparison between clusters J and E. ${ }^{*}$ Comparison between clusters $\mathrm{H}$ and $\mathrm{E}$.

and gram-positive bacteria $[39,40]$. The three mechanisms of such resistance are changes in the ribosomal binding sites of antibiotics, reduced penetration of antibiotics into bacteria, and enzymatic inactivation of antibiotics. Enzymatic inactivation is the most prevalent mechanism of resistance to aminoglycosides [41].

Study findings also showed that $42.8 \%$ of K. pneumoniae isolates were able to form biofilm, and $34.2 \%$ of those with this ability were moderate to strong biofilm producers. Biofilm formation is an important step in the development and stabilization of opportunistic nosocomial VAP because it protects bacteria against phagocytosis. Colonization of VAP-induced bacteria in endotracheal tube and ventilator circuit is very common, and there is a well-known significant relationship between such colonization and nosocomial pneumonia [42]. K. pneumoniae is a major cause of VAP, and biofilm formation is an important pathogenic factor among patients with VAP induced by K. pneumoniae. Biofilm formation ability seems to have direct relationship with environmental resistance among $K$. pneumoniae strains [43]. A former study into the biofilm formation ability of K. pneumoniae reported a biofilm formation rate of $62.5 \%$ [44]. Another study found that $37.6 \%$ of $K$. pneumoniae strains formed biofilm [13]. This difference among studies regarding the rate of biofilm formation by K. pneumoniae is attributable to the differences in the geographical area, setting, and sample size of the studies. Effective management of infections caused by biofilm-producing microorganisms using available antibiotics is essential in healthcare settings. Recently, some novel biofilm-eliminating strategies have 
been developed. Examples of these strategies are use of bacteriophage, weak organic acids, and photo irradiation. Nonetheless, further studies are still needed to produce firmer evidence in this area [45].

MST and dendrogram analysis in this study revealed 28 different ERIC types, namely, ten prevalent types and eighteen unique types. This finding denotes the great diversity of the K. pneumoniae isolates obtained from the study setting. Several previous studies in Iran and other countries also reported genetic diversity among $K$. pneumoniae isolates. For instance, a study on CST-resistant K. pneumoniae isolates in the southwest of Iran reported 23 ERIC types among 26 isolates [46]. Another study reported 32 ERIC types among $35 \mathrm{~K}$. pneumoniae isolates. Moreover, a study into the genetic relatedness of MDRKp isolates in hospitals in Egypt reported 21 ERIC types and great genetic diversity [19]. These contradictory findings are related to the high heterogeneity of pathogenic $K$. pneumoniae due to differences in its nucleotide sequences [47].

Study findings also showed that K. pneumoniae isolates in the largest cluster, i.e., cluster J, had high antimicrobial resistance to antibiotics Mer, CPM, TOB, AZT, GM, CIP, and PTZ, and the most prevalent antimicrobial resistance pattern among them was Mer+CPM+TOB+AZT+IMI+GM $+\mathrm{CIP}+\mathrm{PTZ}$. Antimicrobial resistance to Mer, TOB, GM, and CIP in this cluster was significantly higher than cluster $\mathrm{H}$. Moreover, antimicrobial resistance to TOB in cluster J was significantly higher than cluster E. These findings denote significant relationship between ERIC clusters and antimicrobial resistance. However, there was no significant difference among the three major clusters $\mathrm{E}, \mathrm{H}$, and J respecting biofilm formation. The high diversity of K. pneumoniae isolates in the present study is an important finding because more than $70 \%$ of these isolates are MDR and can use mechanisms such as horizontal gene transfer to transfer this resistance to the bacteria which induce healthcare-associated infections. Different clones may have different antimicrobial resistance patterns and thereby can cause more difficulties in the treatment of their associated infections [22]. Given the significant role of antimicrobial resistance in the management of VAP, the high prevalence of resistant strains can complicate the conditions of patients with COVID-19. Therefore, determination of antibiotic-resistant bacteria, selection of appropriate antibiotics for VAP management, and close adherence to nosocomial infection management guidelines in hospital wards, particularly in ICU, are recommended to reduce mortality rate among these patients.

The rates of VAP between centers managing COVID-19 are likely to vary depending on the clinical characteristics of the patients managed, differential ICU admission policies, and clinical factors such as use of immunosuppressive therapies. However, it is thought that increasing the number of nurses in the ICU, educating nurses about oral hygiene, and head of bed elevation in these centers can help reduce VAP cases.

\section{Conclusion}

This study suggests that K. pneumoniae isolates obtained from patients with COVID-19 have high antimicrobial resis- tance, and the prevalence of MDR strains among them is very high (77\%). Moreover, $42.8 \%$ of these isolates are biofilm producer, and $44.2 \%$ of them have genetic similarity and are clustered in three main clusters with significant differences among clusters respecting genetic similarity and antimicrobial resistance of the isolates. Determining the characteristics of these isolates helps medical specialists more effectively manage their associated infections and use more effective infection management policies for each setting. Further studies are recommended for assessing genetic diversity, ERIC types, and antimicrobial resistance pattern among different bacterial species which cause VAP among patients with COVID-19 in order to facilitate patient recovery. Future studies are needed to assess bacterial colonization among patients with COVID-19 at the time of their hospital admission.

\section{Data Availability}

Data are available on request.

\section{Conflicts of Interest}

Authors declare no conflict of interest.

\section{Authors' Contributions}

MM designed the study, analyzed the data, and wrote the manuscript. AG contributed to sample preparation. MN designed the study. SR and ZM performed the experiments.

\section{Acknowledgments}

This study was supported by the Kashan University of Medical Sciences, Kashan, Iran (grant number: 98122).

\section{References}

[1] M. Maes, J. Pereira-Dias, M. D. Curran et al., "Ventilator-associated pneumonia in critically ill patients with COVID-19," Critical Care, vol. 25, no. 1, p. 25, 2021.

[2] H. C. C. Póvoa, G. C. Chianca, and N. Iorio, "COVID-19: an alert to ventilator-associated bacterial pneumonia," Infectious Disease and Therapy, vol. 9, no. 3, pp. 417-420, 2020.

[3] F. Zhou, T. Yu, R. du et al., "Clinical course and risk factors for mortality of adult inpatients with COVID-19 in Wuhan, China: a retrospective cohort study," Lancet, vol. 395, no. 10229, pp. 1054-1062, 2020.

[4] J. L. Vincent, Y. Sakr, M. Singer et al., "Prevalence and outcomes of infection among patients in intensive care units in 2017," JAMA, vol. 323, no. 15, pp. 1478-1487, 2020.

[5] A. A. Kalanuria, W. Ziai, and M. Mirski, "Ventilator-associated pneumonia in the ICU," Critical Care, vol. 18, no. 2, pp. 208-208, 2014.

[6] S. Kharel, A. Bist, and S. K. Mishra, "Ventilator-associated pneumonia among ICU patients in WHO Southeast Asian region: a systematic review," PLoS One, vol. 16, no. 3, pp. e0247832-e0247832, 2021.

[7] S. Guo, J. J. Xu, Y. S. Wei, J. H. Xu, Y. Li, and R. Xue, "Clinical and molecular characteristics of Klebsiella pneumoniae 
ventilator-associated pneumonia in mainland China," $B M C$ Infectious Diseases, vol. 16, no. 1, p. 608, 2016.

[8] G. Montrucchio, S. Corcione, G. Sales, A. Curtoni, F. G. de Rosa, and L. Brazzi, "Carbapenem-resistant Klebsiella pneumoniae in ICU-admitted COVID-19 patients: Keep an eye on the ball," Journal of Global Antimicrobial Resistance, vol. 23, pp. 398-400, 2020.

[9] J. K. Palmeiro, R. F. de Souza, M. A. Schörner et al., "Molecular epidemiology of multidrug-resistant Klebsiella pneumoniae isolates in a Brazilian tertiary hospital," Frontiers in Microbiology, vol. 10, p. 1669, 2019.

[10] J. M. Munita and C. A. Arias, "Mechanisms of antibiotic resistance," Microbiol Spectr, vol. 4, no. 2, p. 2, 2016.

[11] R. M. Donlan, "Biofilms: microbial life on surfaces," Emerging Infectious Diseases, vol. 8, no. 9, pp. 881-890, 2002.

[12] M. W. LeChevallier, C. D. Cawthon, and R. G. Lee, "Factors promoting survival of bacteria in chlorinated water supplies," Applied and Environmental Microbiology, vol. 54, no. 3, pp. 649-654, 1988.

[13] H. Nirwati, K. Sinanjung, F. Fahrunissa et al., "Biofilm formation and antibiotic resistance of Klebsiella pneumoniae isolated from clinical samples in a tertiary care hospital, Klaten, Indonesia," BMC Proceedings, vol. 13, no. S11, p. 20, 2019.

[14] L. Berra, L. de Marchi, Z. X. Yu, P. Laquerriere, A. Baccarelli, and T. Kolobow, "Endotracheal tubes coated with antiseptics decrease bacterial colonization of the ventilator circuits, lungs, and endotracheal tube," Anesthesiology, vol. 100, no. 6, pp. 1446-1456, 2004.

[15] B. Li, Y. Zhao, C. Liu, Z. Chen, and D. Zhou, "Molecular pathogenesis of Klebsiella pneumoniae," Future Microbiology, vol. 9, no. 9, pp. 1071-1081, 2014.

[16] C. N. Murphy and S. Clegg, "Klebsiella pneumoniae and type 3 fimbriae: nosocomial infection, regulation and biofilm formation," Future Microbiology, vol. 7, no. 8, pp. 991-1002, 2012.

[17] C. Potera, "Forging a link between biofilms and disease," Science, vol. 283, no. 5409, pp. 1837-1839, 1999.

[18] M. Motallebi, Z. Alibolandi, Z. F. Aghmiyuni, W. B. van Leeuwen, M. R. Sharif, and R. Moniri, "Molecular analysis and the toxin, MSCRAMM, and biofilm genes of methicillin- resistant Staphylococcus aureus strains isolated from pemphigus wounds: A study based on SCC mec and dru typing," Infection, Genetics and Evolution, vol. 87, article 104644, 2021.

[19] R. Wasfi, W. F. Elkhatib, and H. M. Ashour, "Molecular typing and virulence analysis of multidrug resistant Klebsiella pneumoniae clinical isolates recovered from Egyptian hospitals," Scientific Reports, vol. 6, no. 1, 2016.

[20] M. Motallebi, F. Jabalameli, R. Beigverdi, and M. Emaneini, "High prevalence of direct repeat unit types of 10di, $8 \mathrm{~h}$ and $8 \mathrm{i}$ among methicillin resistant Staphylococcus aureus strains with staphylococcal cassette chromosome mec type IIIA isolated in Tehran, Iran," Antimicrobial Resistance \& Infection Control, vol. 8, no. 1, pp. 1-8, 2019.

[21] W. Li, D. Raoult, and P.-E. Fournier, "Bacterial strain typing in the genomic era," FEMS Microbiology Reviews, vol. 33, no. 5, pp. 892-916, 2009.

[22] P. Sedighi, O. Zarei, K. Karimi, M. Taheri, P. Karami, and L. Shokoohizadeh, "Molecular typing of Klebsiella pneumoniae clinical isolates by Enterobacterial repetitive intergenic consensus polymerase chain reaction," International Journal of Microbiology, vol. 2020, 5 pages, 2020.
[23] M. Agha-Seyed Hosseini, F. Firoozeh, A. Piroozmand, and H. R. Gilasi, "Carbapenemase-producing Klebsiella pneumoniae strains among clinical specimens in Kashan (20142015)," KAUMS Journal (FEYZ), vol. 20, no. 3, pp. 267-273, 2016.

[24] M. Ali, S. Anwar, N. J. Toma, I. Rafid, M. K. Hasan, and M. J. Foysal, "Molecular detection and PCR-RFLP analysis of Mucoviscosity-associated gene A (magA) in clinical isolates of multidrug-resistant Klebsiella pneumoniae in Bangladesh," The Open Microbiology Journal, vol. 14, no. 1, pp. 196-204, 2020.

[25] Clinical and Laboratory Standards Institute (CLSI), 2019, https://clsi.org/standards/products/microbiology/. (January 2019).

[26] C. Liu and J. Guo, "Characteristics of ventilator-associated pneumonia due to hypervirulent Klebsiella pneumoniae genotype in genetic background for the elderly in two tertiary hospitals in China," Antimicrobial Resistance and Infection Control, vol. 7, no. 1, p. 95, 2018.

[27] S. K. Shukla and T. S. Rao, An improved crystal violet assay for biofilm quantification in 96-well microtitre plate, no. article 100214, 2017bioRxiv, 2017.

[28] S. Purighalla, S. Esakimuthu, M. Reddy, G. K. Varghese, V. S. Richard, and V. K. Sambandamurthy, "Discriminatory Power of Three Typing Techniques in Determining Relatedness of Nosocomial_Klebsiella pneumoniae_ Isolates from a Tertiary Hospital in India," Indian Journal of Medical Microbiology, vol. 35, no. 3, pp. 361-368, 2017.

[29] J. Versalovic, T. Koeuth, and J. R. Lupski, "Distribution of repetitive DNA sequences in eubacteria and application to fingerprinting of bacterial genomes," Nucleic Acids Research, vol. 19, no. 24, pp. 6823-6831, 1991.

[30] K. Ahmad, M. Mitra, and R. Mehdi, "Characterization of virulence genes in typical and atypical enteropathogenic Escherichia coli," Scientific Research and Essays, vol. 6, no. 31, pp. 6600-6605, 2011.

[31] A. S. Moini, B. Soltani, A. Taghavi Ardakani et al., "Multidrugresistant Escherichia coli and Klebsiella pneumoniae isolated from patients in Kashan, Iran," Jundishapur Journal Of Microbiology, vol. 8, no. 10, pp. e27517-e27517, 2015.

[32] A. Khorshidi, M. Rohani, and R. Moniri, "The prevalence and molecular characterization of extended-spectrum $\beta$-lactamases-producing Klebsiella pneumoniae isolates recovered from Kashan hospital university, Iran," Jundishapur Journal of Microbiology, vol. 4, no. 4, pp. 286-294, 2011.

[33] R. L. Ferreira, B. da Silva, G. S. Rezende et al., "High prevalence of multidrug-resistant Klebsiella pneumoniae harboring several virulence and $\beta$-lactamase encoding genes in a Brazilian intensive care unit," Frontiers in Microbiology, vol. 9, p. 3198, 2018.

[34] K. Rothe, S. Feihl, J. Schneider et al., "Rates of bacterial coinfections and antimicrobial use in COVID-19 patients: a retrospective cohort study in light of antibiotic stewardship," European Journal of Clinical Microbiology \& Infectious Diseases, vol. 40, no. 4, pp. 859-869, 2021.

[35] M. M. Molla, M. Yeasmin, M. K. Islam et al., "Antibiotic prescribing patterns at COVID-19 dedicated wards in Bangladesh: findings from a single center study," Infection Prevention in Practice, vol. 3, no. 2, pp. 100134-100134, 2021.

[36] G. Meletis, "Carbapenem resistance: overview of the problem and future perspectives," Therapeutic Advances in Infectious Disease, vol. 3, no. 1, pp. 15-21, 2016. 
[37] A. Fàbrega, S. Madurga, E. Giralt, and J. Vila, "Mechanism of action of and resistance to quinolones," Microbial Biotechnology, vol. 2, no. 1, pp. 40-61, 2009.

[38] S. M. Hamed, W. F. Elkhatib, H. A. el-Mahallawy, M. M. Helmy, M. S. Ashour, and K. M. A. Aboshanab, "Multiple mechanisms contributing to ciprofloxacin resistance among Gram negative bacteria causing infections to cancer patients," Scientific Reports, vol. 8, no. 1, p. 12268, 2018.

[39] S. Garneau-Tsodikova and K. J. Labby, "Mechanisms of resistance to aminoglycoside antibiotics: overview and perspectives," MedChemComm, vol. 7, no. 1, pp. 11-27, 2016.

[40] M. Laws, A. Shaaban, and K. M. Rahman, "Antibiotic resistance breakers: current approaches and future directions," FEMS Microbiology Reviews, vol. 43, no. 5, pp. 490-516, 2019.

[41] G. Kapoor, S. Saigal, and A. Elongavan, "Action and resistance mechanisms of antibiotics: a guide for clinicians," Journal Of Anaesthesiology, Clinical Pharmacology, vol. 33, no. 3, pp. 300-305, 2017.

[42] O. Diaconu, I. Siriopol, L. I. Poloșanu, and I. Grigoraş, "Endotracheal tube biofilm and its impact on the pathogenesis of ventilator-associated pneumonia," Journal of critical care medicine (Universitatea de Medicina si Farmacie din TarguMures), vol. 4, no. 2, pp. 50-55, 2018.

[43] R. M. Martin and M. A. Bachman, "Colonization, infection, and the accessory genome of Klebsiella pneumoniae," Frontiers in Cellular and Infection Microbiology, vol. 8, p. 4, 2018.

[44] D. Yang and Z. Zhang, "Biofilm-forming Klebsiella pneumoniae strains have greater likelihood of producing extendedspectrum $\beta$-lactamases," The Journal of Hospital Infection, vol. 68 , no. 4, pp. 369-371, 2008.

[45] G. Hughes and M. A. Webber, "Novel approaches to the treatment of bacterial biofilm infections," British Journal of Pharmacology, vol. 174, no. 14, pp. 2237-2246, 2017.

[46] M. Moosavian and N. Emam, "The first report of emerging mobilized colistin-resistance (mcr) genes and ERIC-PCR typing in Escherichia coli and Klebsiella pneumoniae clinical isolates in southwest Iran," Infect Drug Resist, vol. Volume 12, pp. 1001-1010, 2019.

[47] L.-C. Ma, C. T. Fang, C. Z. Lee, C. T. Shun, and J. T. Wang, "Genomic heterogeneity in Klebsiella pneumoniae strains is associated with primary pyogenic liver abscess and metastatic infection," The Journal of Infectious Diseases, vol. 192, no. 1, pp. 117-128, 2005. 